\title{
Hallazgos clínicos y epidemiológicos en la neurofibromatosis tipo 1 y el complejo esclerosis tuberosa en una serie de pacientes pediátricos
}

\author{
Francisco Cammarata-Scalisi ${ }^{1 *}$, Frances Stock², Nicole Velazco ${ }^{3}$, Gloria Da Silva ${ }^{1}$, \\ $M^{a}$ Angelina Lacruz-Rengel ${ }^{4}$ y Andrea Avendaño ${ }^{1}$ \\ ${ }^{1}$ Unidad de Genética Médica, Departamento de Puericultura y Pediatría, Facultad de Medicina, Universidad de Los Andes; ${ }^{2}$ Unidad de Oncología \\ Pediátrica, Instituto Autónomo Hospital Universitario de Los Andes; ${ }^{3}$ U.E. Fundación Colegio Monseñor Bosset; ${ }^{4}$ Servicio de Neuropediatría, \\ Departamento de Puericultura y Pediatría, Facultad de Medicina, Universidad de Los Andes. Mérida, Venezuela
}

\begin{abstract}
Resumen
Introducción: La neurofibromatosis tipo 1 (NF1) es una entidad genética con una incidencia de 1 entre 2,500 a 3,500 nacimientos. Por su parte, el complejo esclerosis tuberosa (CET) presenta una incidencia de 1 entre 6,000 a 10,000 nacimientos. Ambas entidades neurocutáneas cursan con un patrón de herencia autosómico dominante, expresividad variable y la morbimortalidad se encuentra asociada a complicaciones multisistémicas. El objetivo de este trabajo fue exponer las características clínicas y epidemiológicas de una serie de pacientes pediátricos con diagnóstico de NF1 y CET atendidos en la Unidad de Genética Médica de la Universidad de Los Andes. Métodos: Este trabajo corresponde a una serie de casos de pacientes menores de 16 años atendidos en un período de 11 años, que cumplan con los criterios diagnósticos de NF1 y CET según los consensos para cada entidad. Resultados: Se estudiaron 89 pacientes, 73 con NF1 y 16 con CET. Presentaron dos criterios para NF1, 58 (79.45\%) pacientes, y las máculas café con leche fueron las más frecuentes y presentes en todos los casos; 10 pacientes $(62.50 \%)$ presentaron dos criterios mayores para el CET, y las máculas hipocrómicas estuvieron igualmente presentes en todos los casos. Conclusiones: Este estudio muestra la forma de presentación clínica de las dos entidades neurocutáneas más frecuentes. Se discuten los criterios diagnósticos con el objeto de identificarlos a edades más tempranas y poder brindar una evaluación médica interdisciplinaria, tratamiento y un oportuno asesoramiento genético familiar.
\end{abstract}

Palabras clave: Neurofibromatosis tipo 1. Complejo esclerosis tuberosa. Clínica. Consensos diagnósticos. Diagnóstico temprano.

\section{Clinical and epidemiological findings in neurofibromatosis type 1 and tuberous sclerosis complex in a series of pediatric patients}

\begin{abstract}
Background: Neurofibromatosis type 1 (NF1) is a genetic entity with an incidence of 1 in 2,500 to 3,500 births. Tuberous sclerosis complex (TSC) has an incidence between 1 in 6,000 to 10,000 births. Both neurocutaneous entities present an autosomal dominant inheritance pattern, variable expressivity and their morbidity and mortality is associated with multisystemic
\end{abstract}

Correspondencia:

*Francisco Cammarata Scalisi

E-mail: francocammarata19@gmail.com
Disponible en internet: 25-09-2018 Bol Med Hosp Infant Mex. 2018:75:287-294 www.bmhim.com 1665-1146/@ 2018. Hospital Infantil de México Federico Gómez, impreso por Permanyer México SA de CV, todos los derechos reservados. 
complications. The aim of this study was to present the clinical and epidemiological characteristics of a series of pediatric patients diagnosed with NF1 and TSC, who were treated in the Medical Genetics Unit of the Universidad of Los Andes. Methods: This work corresponds to a series of cases of patients under 16 years of age served in a period of 11 years, who met the diagnostic criteria of NF1 and CET according to the consensus for each entity. Results: We studied 89 patients, 73 with NF1 and 16 with TSC. 58 (79.45\%) of the patients presented two criteria for NF1, with café-au-lait macules being the most frequent and present in all cases. 10 (62.50\%) of the patients presented two major criteria for TSC; hypochromic macules were equally present in all cases. Conclusions: This study shows the clinical presentation of the two most frequent neurocutaneous entities. Diagnostic criteria are discussed in order to perform them at younger ages and to provide an interdisciplinary medical evaluation, treatment and timely family genetic counseling.

Key words: Neurofibromatosis type 1. Tuberous sclerosis complex. Clinic. Diagnostic consensus. Early diagnosis.

\section{Introducción}

La neurofibromatosis tipo 1 (NF1; OMIM 162200) o enfermedad de von Recklinghausen es una entidad genética ${ }^{1}$, con patrón de herencia autosómico dominante ${ }^{2-4}$, que presenta una incidencia de 1 entre 2,500 a 3,500 nacimientos $2,4,5$. Este síndrome se caracteriza clínicamente por cursar máculas café con leche, efélides en la región axilar e inguinal ${ }^{1,3,5,6}$, nódulos de Lisch en el iris (hamartomas) ${ }^{1,5,6}$, glioma en el nervio óptico ${ }^{6}$, neurofibromas cutáneos y plexiforme $e^{1,3,5,6}$ anomalías esqueléticas ${ }^{3,5,6}$ y mayor riesgo de desarrollo de tumores $^{1,3}$. También se puede presentar déficit cognitivo², dificultad en el aprendizaje ${ }^{2,3,5,7}$, trastorno en el patrón de sueño, epilepsia, macrocefalia y alteraciones en la neuroimagen ${ }^{7}$. La NF1 cursa con expresividad variable $^{1,4,6}$, incluso en gemelos monocigóticos ${ }^{8}$, con casi el $100 \%$ de penetrancia ${ }^{6}$, y la morbimortalidad está asociada a complicaciones multisistémicas ${ }^{1}$, las cuales no son predecibles ${ }^{6}$. Los signos clínicos diagnósticos suelen aparecer durante la infancia y la pubertad ${ }^{3}$, por lo que son dependientes de la edad ${ }^{9}$, y el diagnóstico puede verse retrasado a edades tempranas. Es causada por mutaciones heterocigotas en el gen NF1 (OMIM 613113), un gen supresor de tumor que codifica una proteína denominada neurofibromina, que se encuentra en $17 q 11.2^{3,4,6,9,10}$, con aparición de novo en aproximadamente el 50 a $60 \%$ de los $\operatorname{casos}^{4,7,10}$, ya que presenta el índice de mutaciones espontáneas más alto de todo el genoma ${ }^{9}$. A pesar de ser el trastorno neurocutáneo más frecuente ${ }^{1,5}$, el número de trabajos con un número amplio de niños son escasos ${ }^{1}$.

Por su parte, el complejo esclerosis tuberosa (CET OMIM 191100 y 613254) es igualmente un trastorno multisistémico con patrón de herencia autosómico dominante ${ }^{11-15}$. Se caracteriza por la presencia de crecimiento de hamartomas en el cerebro, ojos, piel, riñones, corazón, pulmón e hígado ${ }^{11,12,14}$, con aumento del riesgo de malignidad ${ }^{15}$. Se puede presentar, además, retraso mental, el cual está asociado con el genotipo, los túberes corticales y el síndrome convulsivo ${ }^{13}$. Estas alteraciones constituyen la principal causa de morbimortalidad $^{15}$, además del trastorno del espectro autista ${ }^{16}$, entre otras alteraciones neurológicas. EI CET cursa con expresividad variable, incluso en gemelos monocigóticos afectados, y presenta una penetrancia casi completa ${ }^{11}$. La incidencia del CET oscila de 1 entre 6,000 a 10,000 nacimientos ${ }^{14,15}$, y la mayoría ocurren de forma esporádica, con historia familiar en el 30\% de los casos ${ }^{12,14}$. Es causado por mutaciones heterocigóticas en los genes supresores de tumor TSC1 (OMIM 605284), localizado en 9q34.13, que codifica a la hamartina, y el TSC2 (OMIM 191092), localizado en 16p13.3, que codifica a la tuberina $11,12,14,15$. Las mutaciones en estos genes se detectan en aproximadamente el 85 a $90 \%$ de los casos. Ambas proteínas (TSC1 y TSC2) juegan un rol en la regulación de metabolismo celular, proliferación y diferenciación, al participar en la estructura y función de un complejo multiproteico citosólico, que es un inhibidor de señalización mTOR1 (por sus siglas en inglés, mammalian target of rapamycin), por lo que actúan como supresores de tumor ${ }^{17}$.

El objetivo del presente estudio es exponer las características clínicas y epidemiológicas de una serie de pacientes pediátricos con diagnóstico de NF1 y CET atendidos en la Unidad de Genética Médica de la Universidad de Los Andes (UGM-ULA).

\section{Métodos}

El presente trabajo consiste en una serie de casos, en que se analizaron los datos clínicos y epidemiológicos disponibles de pacientes menores de 16 años atendidos en la consulta de la UGM-ULA entre enero de 2007 y diciembre de 2017. 
Tabla 1. Criterios clínicos diagnósticos de neurofibromatosis tipo $1^{1,4,10}$

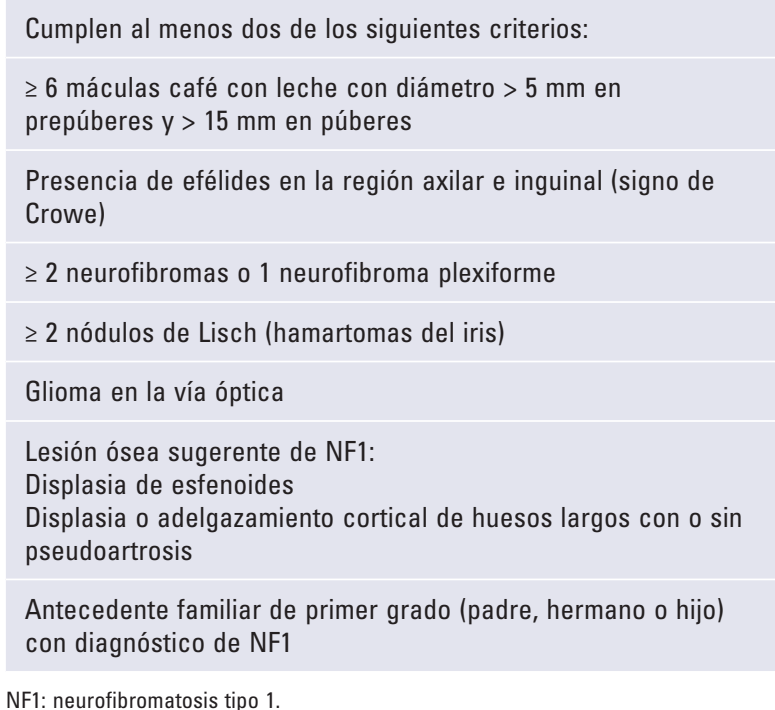

NF1: neurofibromatosis tipo 1.

Se incluyeron en el estudio pacientes con diagnóstico de NF1, de acuerdo con los criterios del Instituto Nacional de Salud de los EE.UU. (Tabla 1) 1,4,10. Igualmente, se incluyeron los pacientes con diagnóstico definitivo de CET, según el Grupo Internacional de la entidad actualizado en el 2012 (Tabla 2) 14,18. Por lo tanto, se excluyeron aquellos pacientes que no reunieron los criterios clínicos mínimos para establecer dichos diagnósticos.

\section{Resultados}

Se analizaron 89 pacientes, 73 con NF1 y 16 con $\mathrm{CET}$, que cumplieron los criterios de inclusión. De los pacientes menores de 8 años de edad, 41 (56.16\%) presentaron NF1 y 8 (50\%) CET (Tabla 3).

De los pacientes incluidos, 58 (79.45\%) presentaron dos criterios que definieron el diagnóstico de NF1, $14(19.18 \%)$ presentaron tres criterios y uno (1.37\%) presentó cuatro. Las máculas hipercrómicas estuvieron presentes en la totalidad de los casos, y las efélides en la región axilar e inguinal se presentaron en 29 pacientes (90.63\%) del grupo de 8 a 15 años (Tabla 4). Los hallazgos adicionales encontrados se muestran en la tabla 5.

Diez $(62.50 \%)$ pacientes presentaron dos criterios mayores para el diagnóstico de CET, cuatro $(25 \%)$ presentaron tres criterios, un paciente $(6.25 \%)$ presentó cinco criterios y también un paciente (6.25\%) presentó siete criterios. Las máculas hipocrómicas estuvieron presentes en la totalidad de los casos; las displasias

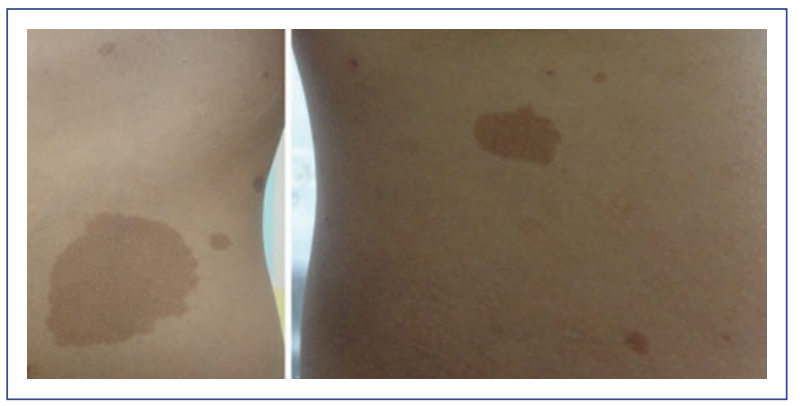

Figura 1. Múltiples máculas café con leche en un segmento corporal en pacientes pediátricos con NF1.

corticales y angiofibromas se detectaron en 5 (62.5\%) pacientes, en el grupo de menores de 8 años (Tabla 6 ).

\section{Discusión}

La NF1 y el CET presentan un patrón de herencia autosómico dominante, por lo que ambos sexos se encuentran afectados por igual; en este trabajo se demostró la distribución por sexos. La mayoría de los pacientes estudiados son el primer caso en la familia, como se muestra en la tabla 3. Como ya se mencionó, la presencia de antecedente familiar de primer grado constituye un criterio clínico diagnóstico de NF1 (Tabla 1); sin embargo, este criterio no es característico en el CET.

Los criterios clínicos diagnósticos para la NF1 son bastante sensibles y específicos en adultos, no así en niños menores de 8 años ${ }^{4,9}$. Además, no tienen en cuenta otros síntomas como la dificultad de aprendizaje, macrocefalia ${ }^{7,9}$ o la presencia de neoplasias, como el tumor maligno de la vaina de los nervios periféricos ${ }^{9}$. En un estudio en 1,893 pacientes menores de 21 años con NF1, se encontró que el $46 \%$ de los pacientes eran el primer caso en la familia y no cumplían criterios diagnósticos antes del primer año de edad, mientras que a los 8 años los cumplían el $97 \%$ y a los 20 años, el 100\% de los $\operatorname{casos}^{9,19}$. Este aspecto se evidenció en el presente estudio, en el cual fueron excluidos nueve lactantes que presentaron solamente máculas café con leche sin ningún otro criterio; sin embargo, estos pacientes se mantienen en seguimiento de evolución del fenotipo.

Las máculas café con leche constituyen el hallazgo clínico más frecuente ${ }^{9,10}$ y precoz de NF1, y pueden ser congénitas o aparecer a lo largo del primer año de vida (99\% de los casos) y aumentar en número durante la infancia. Estas lesiones no se presentan en el cuero cabelludo, palmas ni plantas, y son de color y tamaño variable, incluso en un mismo individuo ${ }^{9}$ (Fig. 1); sin embargo, aunque son sugestivas, no son 
Tabla 2. Criterios diagnósticos actualizados para el complejo esclerosis tuberosa $2012^{14,17}$

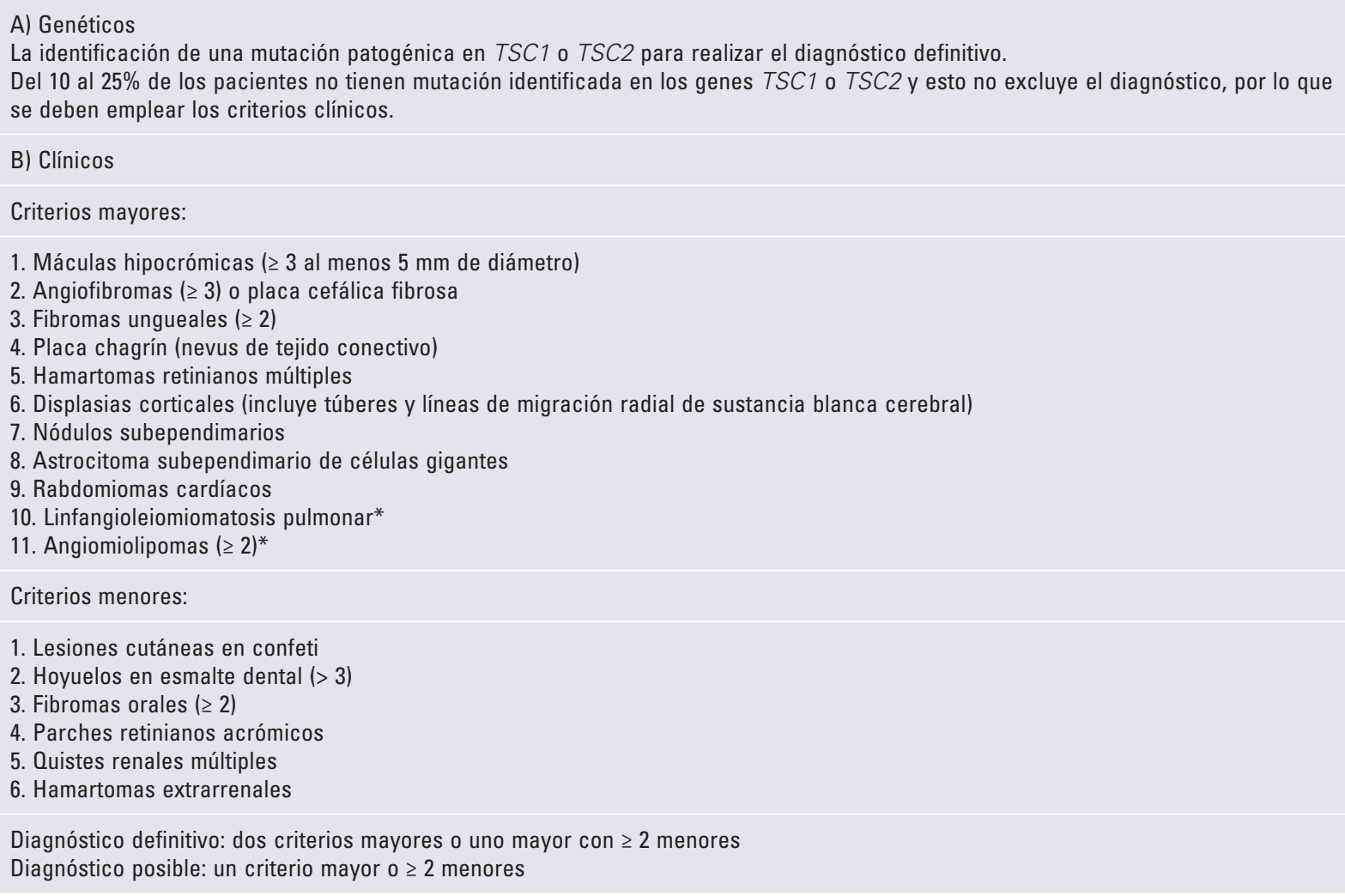

*Una combinación de estos dos criterios mayores sin otros hallazgos no cumplen con el criterio de diagnóstico definitivo.

Tabla 3. Características epidemiológicas de los pacientes estudiados con neurofibromatosis tipo 1 y complejo esclerosis tuberosa

\begin{tabular}{|c|c|c|c|c|c|c|}
\hline \multirow[t]{3}{*}{ Características/entidades } & \multicolumn{2}{|c|}{ NF1 } & \multirow[t]{3}{*}{ Total } & \multicolumn{2}{|c|}{ CET } & \multirow[t]{3}{*}{ Total } \\
\hline & $<8$ años & 8 a 15 años & & $<8$ años & 8 a 15 años & \\
\hline & $n(\%)$ & n (\%) & & n $(\%)$ & n (\%) & \\
\hline $\begin{array}{l}\text { Sexo } \\
\text { Femenino } \\
\text { Masculino }\end{array}$ & $\begin{array}{l}23(56.10) \\
18(43.90)\end{array}$ & $\begin{array}{l}17(53.13) \\
15(46.87)\end{array}$ & $\begin{array}{l}40 \\
33\end{array}$ & $\begin{array}{l}5(62.5) \\
3(37.5)\end{array}$ & $\begin{array}{l}3(37.5) \\
5(62.5)\end{array}$ & $\begin{array}{l}8 \\
8\end{array}$ \\
\hline $\begin{array}{l}\text { Procedencia por estados } \\
\text { Mérida } \\
\text { Barinas } \\
\text { Zulia } \\
\text { Táchira } \\
\text { Trujillo }\end{array}$ & $\begin{array}{c}33(80.49) \\
4(9.76) \\
3(7.31) \\
1(2.44) \\
-\end{array}$ & $\begin{array}{c}27(84.38) \\
2(6.25) \\
1(3.12) \\
1(3.12) \\
1(3.12)\end{array}$ & $\begin{array}{c}60 \\
6 \\
4 \\
2 \\
1\end{array}$ & $\begin{array}{c}7(87.5) \\
- \\
- \\
1(12.5) \\
-\end{array}$ & $\begin{array}{c}6(75) \\
- \\
1(12.5) \\
- \\
1(12.5)\end{array}$ & $\begin{array}{l}13 \\
- \\
1 \\
1 \\
1\end{array}$ \\
\hline Antecedentes familiares de la entidad* & $18(43.90)$ & $7(21.88)$ & 25 & $1(12.5)$ & - & 1 \\
\hline Sin antecedentes & $23(56.10)$ & $25(78.12)$ & 48 & $7(87.5)$ & $8(100)$ & 15 \\
\hline
\end{tabular}

NF1: neurofibromatosis tipo 1; CET: complejo esclerosis tuberosa.

*En la Figura 3, se muestra un ejemplo de diversos familiares afectados con NF1.

patognomónicas de la entidad. Hasta el $20 \%$ de los niños presentan una lesión hipercrómica aislada, el $4 \%$ tienen dos y menos del $1 \%$ de la población sana tiene más de tres (a estos pacientes se les debe realizar un seguimiento riguroso). Entre los diagnósticos diferenciales se encuentran nevus hipercrómicos, mosaicismos pigmentarios, nevus melanocíticos congénitos, nevus spilus, urticaria pigmentosa macular 0 
Tabla 4. Frecuencia de los criterios clínicos diagnósticos en los pacientes con neurofibromatosis tipo 1

\begin{tabular}{|l|c|c|c|c|c|c|}
\hline \multirow{2}{*}{ Criterios clínicos diagnósticos } & \multicolumn{2}{|c|}{$<\mathbf{8}$ años } & \multicolumn{2}{|c|}{$\mathbf{8}$ a 15 años } & \multicolumn{3}{|c|}{ Total } \\
\cline { 2 - 7 } & $\mathbf{n}$ & $\%$ & $\mathbf{n}$ & $\%$ & $\mathbf{n}$ & 100 \\
\hline Máculas hipercrómicas & 41 & 100 & 32 & 100 & 73 & 54.79 \\
\hline Efélides en la región axilar e inguinal & 11 & 26.83 & 29 & 90.63 & 40 & 25 \\
\hline Familiar de primer grado & 21 & 51.22 & 4 & 12.5 & 34.25 \\
\hline Neurofibromas & 2 & 4.88 & 13 & 40.63 & 15 & 20.55 \\
\hline Nódulos de Lisch & 1 & 2.44 & 6 & 18.75 & 7 & 9.59 \\
\hline Glioma del nervio óptico & 1 & 2.44 & 1 & 3.13 & 2 & 2.74 \\
\hline Neurofibroma plexiforme & - & - & 1 & 3.13 & 1 & 1.37 \\
\hline
\end{tabular}

Tabla 5. Hallazgos adicionales encontrados en los pacientes con neurofibromatosis tipo 1

\begin{tabular}{|c|c|c|c|c|}
\hline Otros hallazgos clínicos & $<8$ años & 8 a 15 años & Total & $\%$ \\
\hline \multicolumn{5}{|l|}{ Neurológicos } \\
\hline Retraso psicomotor & 6 & 5 & 11 & 15.07 \\
\hline Dificultad en el aprendizaje & 3 & 6 & 9 & 12.33 \\
\hline Síndrome convulsivo & 4 & 2 & 6 & 8.22 \\
\hline Trastorno de hiperactividad con déficit de atención & 1 & 5 & 6 & 8.22 \\
\hline Retraso en el lenguaje & 2 & 3 & 5 & 6.85 \\
\hline Autismo & - & 1 & 1 & 1.37 \\
\hline Mielomeningocele lumbar & 1 & - & 1 & 1.37 \\
\hline Cuadriparesia espástica & 1 & - & 1 & 1.37 \\
\hline \multicolumn{5}{|l|}{ Esqueléticos } \\
\hline Escoliosis & 5 & 4 & 9 & 12.33 \\
\hline Hiperlordosis lumbar & - & 2 & 2 & 2.74 \\
\hline Retraso pondoestatural & 2 & 4 & 6 & 8.22 \\
\hline Neoplasias & 3 & 2 & 5 & 6.85 \\
\hline Gastrointestinal: enfermedad de Hirschsprung & 1 & - & 1 & 1.37 \\
\hline
\end{tabular}

hiperpigmentación postinflamatoria ${ }^{20}$. Las máculas café con leche estuvieron presentes en todos los pacientes analizados en este estudio (Tabla 4) y constituyeron, sin duda, el principal motivo de consulta y causa del seguimiento clínico desde los primeros meses de vida.

Las efélides en la región axilar e inguinal, también conocidas como signo de Crowe, se observan generalmente entre el tercer y el quinto año de edad, y constituyen el hallazgo cutáneo más específico y, para algunos autores, casi patognomónico ${ }^{9}$.En nuestro estudio, este hallazgo estuvo presente en 29 (90.63\%) pacientes en el grupo de 8 a 15 años con NF1, como se muestra en la tabla 4. Por su parte, los neurofibromas pueden aparecer en cualquier parte del cuerpo, generalmente después de la pubertad ${ }^{9}$. En nuestro estudio fue el cuarto criterio en pacientes con NF1, posterior al antecedente familiar de primer grado afectado con este síndrome (Tabla 4).

Se está considerando la importancia de los nevus anémicos y el xantogranuloma juvenil ${ }^{1,9}$ como criterios diagnósticos ante su asociación evidente y presentación 
Tabla 6. Frecuencia de los criterios clínicos diagnósticos en los pacientes con complejo esclerosis tuberosa

\begin{tabular}{|l|c|c|c|c|c|c|}
\hline \multirow{2}{*}{ Criterios clínicos diagnósticos } & \multicolumn{2}{|c|}{$<\mathbf{8}$ años } & \multicolumn{2}{|c|}{$\mathbf{8}$ a 15 años } & \multicolumn{3}{c|}{ Total } \\
\cline { 2 - 7 } & $\mathbf{n}$ & $\%$ & $\mathbf{n}$ & $\%$ & $\mathbf{n}$ & $\%$ \\
\hline Máculas hipocrómicas & 8 & 100 & 8 & 100 & 16 & 100 \\
\hline Displasias corticales & 5 & 62.5 & 4 & 50 & 9 & 56.25 \\
\hline Angiofibromas & 5 & 62.5 & 3 & 37.5 & 8 & 50 \\
\hline Nódulos subependimarios & 2 & 25 & 2 & 25 & 4 & 25 \\
\hline Astrocitoma subependimario de células gigantes & - & - & 2 & 25 & 2 & 12.5 \\
\hline Placa chagrín & - & - & 2 & 25 & 2 & 12.5 \\
\hline Fibromas ungueales & 1 & 12.5 & 1 & 12.5 & 2 & 12.5 \\
\hline Rabdomiomas cardíacos & 1 & 12.5 & - & - & 1 & 6.25 \\
\hline
\end{tabular}

temprana, ya que en niños menores de dos años y primer caso de NF1 en la familia se retardaría el diagnóstico'.

Los pacientes con NF1 pueden desarrollar complicaciones relacionadas con los neurofibromas plexiformes, con un riesgo aumentado de malignización hasta en un tercio de los $\operatorname{casos}^{7}$.Se ha descrito que del 8 al $13 \%$ de los casos de este síndrome pueden desarrollar dicho tumor maligno de la vaina de los nervios periféricos en la edad adulta ${ }^{4,9}$, así como otros tipos de neoplasias en el sistema nervioso y leucemia mielomonocítica juvenil21 ${ }^{21}$ Es por ello que todo paciente pediátrico con diagnóstico o sospecha de NF1 sigue un esquema interdisciplinario de controles, además de evaluación por el servicio de oncología. Igualmente, se deben incluir dentro los criterios diagnósticos de NF1 la presencia de neoplasias.

La dificultad en el aprendizaje $e^{7,10,22,23}$ y la alteración en el comportamiento se presentan con mayor frecuencia en la NF1 que en la población general, asociadas con, entre otras alteraciones neurológicas, el retraso mental ${ }^{10,22,23}$, la dificultad en la coordinación motora ${ }^{10}$ y en el lenguaje ${ }^{10,23}$, el trastorno por déficit de atención con hiperactividad ${ }^{10,22,23}$,el autismo ${ }^{22,23}$ y la migraña ${ }^{22}$. Algunos de estos hallazgos se muestran en la tabla 5 . Es probable que exista un subregistro al no representar criterios diagnósticos de la NF1, dado que los datos se obtuvieron de la historia clínica de la UGM-ULA y no de todos los servicios de interconsulta. Del mismo modo, el diagnóstico de algunas de estas alteraciones neurológicas no se realiza en etapas tempranas del desarrollo.

Por otra parte, el diagnóstico clínico del CET se basa en la identificación de criterios diagnósticos mayores y

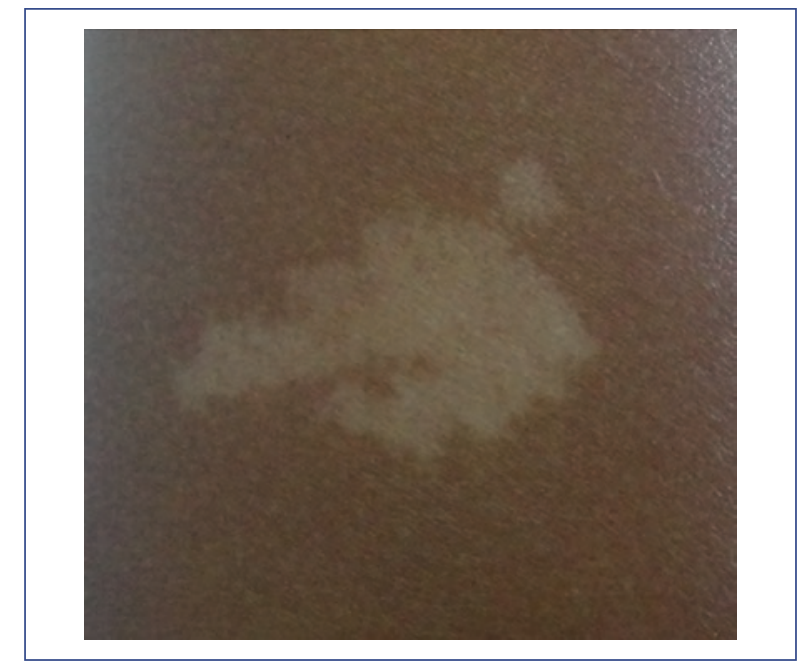

Figura 2. Mácula hipocrómica en el CET.

menores ya descritos ${ }^{14,18}$. Las máculas hipocrómicas estuvieron presentes en todos los pacientes evaluados en este trabajo (Fig. 2), seguidas de las displasias corticales en nueve pacientes (56.25\%) (Tabla 6). Solo en un caso se detectó la presencia de rabdomiomas cardíacos, a diferencia de otros estudios donde dicho hallazgo fue el segundo más frecuente $(70 \%)^{24}$, por lo que se recomienda en casos familiares o en los casos en los que se conoce el diagnóstico, la detección prenatal de esta alteración, necesaria para el seguimiento del CET.

A pesar de los avances en el CET, todavía quedan muchas acciones por hacer: la amplia presentación clínica a nivel neurológico, la identificación temprana con el objeto de brindar una vigilancia apropiada, la orientación anticipada y un tratamiento efectivo que 


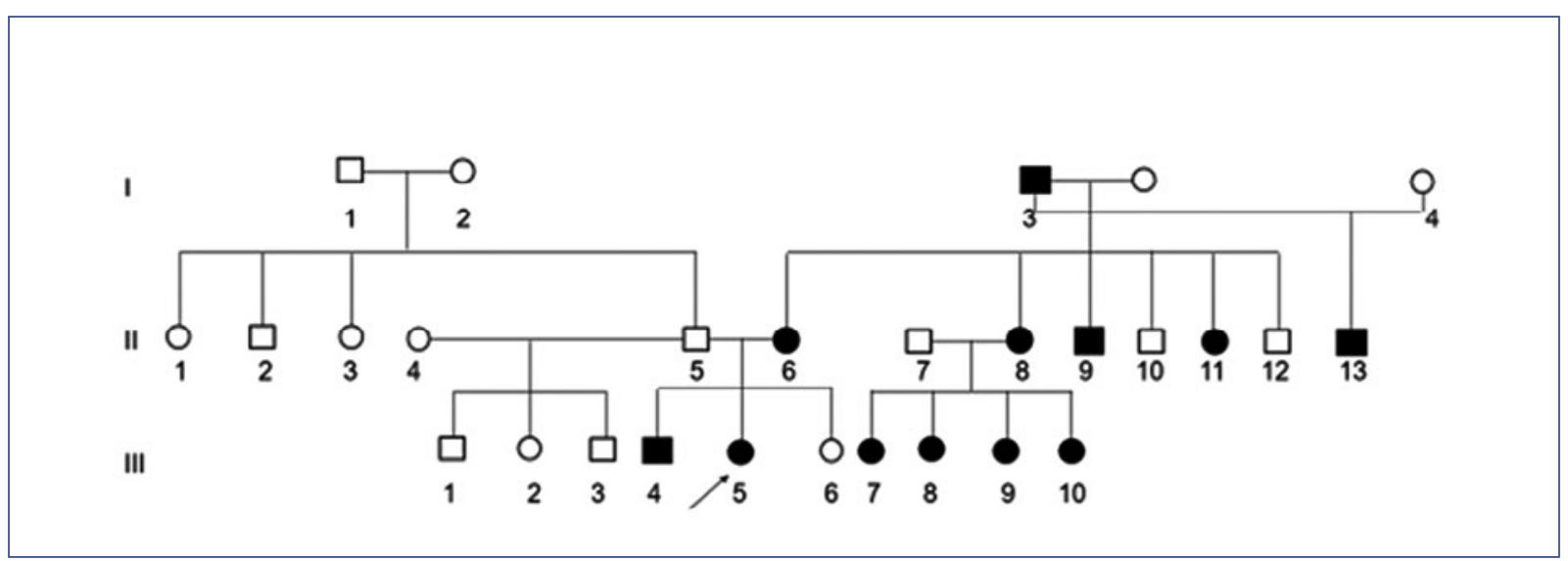

Figura 3. Genealogía que muestra una entidad con patrón de herencia autosómica dominante como es la NF1, donde ambos sexos se encuentran afectados. En todas las generaciones hay personas con la entidad y se puede observar incluso la trasmisión de varón a varón (padre e hijo de sexo masculino afectados). El caso índice en esta familia fue III:5 (se señala con la flecha).

modifique el curso y su pronóstico. Aunque el 90\% de los afectados pueden desarrollar epilepsia y esta puede ser un síntoma inicial ${ }^{25}$, dicho trastorno no se encuentra citado entre los criterios diagnósticos del CET.

El protocolo diagnóstico-terapéutico interdisciplinario del CET precisa de coordinación entre múltiples especialidades pediátricas y del adulto: Obstetricia, Neurología, Psiquiatría, Oftalmología, Odontología, Cardiología, Neumología, Gastropediatría, Nefrología, Urología, Dermatología, Oncología, Radiología y Genética Médica ${ }^{26}$.

Este estudio muestra los diferentes hallazgos clínicos encontrados en NF1 y CET, dos entidades neurocutáneas con presentación más frecuente en la práctica clínica, haciendo énfasis en su forma de presentación. Además, se discuten los criterios diagnósticos, que deben ser periódicamente revisados con el objeto de aumentar la detección a edades más tempranas y brindar una evaluación médica interdisciplinaria y un oportuno asesoramiento genético familiar, ya sea en los casos nuevos o en los familiares (Fig. 3), sabiendo que existe un riesgo del $50 \%$ de que la NF1 o el CET se presenten en el descendiente de algún afectado.

\section{Agradecimientos}

A la Licenciada Rosalia Gumina F, Directora de la Biblioteca del Instituto Autónomo Hospital Universitario de Los Andes, Universidad de Los Andes.

\section{Conflicto de intereses}

Los autores declaran no tener ningún conflicto de intereses.

\section{Fuente financiación}

Ninguna

\section{Responsabilidades éticas}

Protección de personas y animales. Los autores declaran que para esta investigación no se han realizado experimentos en seres humanos ni en animales.

Confidencialidad de los datos. Los autores declaran que han seguido los protocolos de su centro de trabajo sobre la publicación de datos de pacientes.

Derecho a la privacidad y consentimiento informado. Los autores han obtenido el consentimiento informado de los pacientes y/o sujetos referidos en el artículo. Este documento obra en poder del autor de correspondencia.

\section{Bibliografía}

1. Duat Rodríguez A, Martos Moreno GÁ, Martín Santo-Domingo Y, Hernández Martín A, Espejo-Saavedra Roca JM, Ruiz-Falcó Rojas ML, et al. Phenotypic and genetic features in neurofibromatosis type 1 in children. An Pediatr (Barc). 2015;83:173-82.

2. Chambers MA, Miller DT, Ullrich NJ. School liaison program supporting children with neurofibromatosis type 1: a model of care for children with chronic disease. Genet Med. 2017. doi: 10.1038/gim.2017.177.

3. Kallionpää RA, Uusitalo E, Leppävirta J, Pöyhönen M, Peltonen S, Peltonen J. Prevalence of neurofibromatosis type 1 in the Finnish population. Genet Med. 2017. doi: 10.1038/gim.2017.215.

4. Gómez M, Batista O. Molecular diagnosis as a strategy for differential diagnosis and at early ages of neurofibromatosis type 1 (NF1). Rev Med Chil. 2015;143:1320-30.

5. Garcia-Penas JJ. Learning disorders in neurofibromatosis type 1. Rev Neurol. 2017;64:S59-S63.

6. Ehara Y, Yamamoto O, Kosaki K, Yoshida Y. Clinical severity in Japanese patients with neurofibromatosis 1 based on DNB classification. J Dermatol. 2017;44:1262-7.

7. Maraña Pérez Al, Duat Rodríguez A, Soto Insuga V, Domínguez Carral J, Puertas Martín V, González Gutiérrez Solana L. Prevalence of sleep disorders in patients with neurofibromatosis type 1. Neurologia. 2015;30:561-5. 
8. Sites ER, Smolarek TA, Martin LJ, Viskochil DH, Stevenson DA, Ullrich NJ, et al. Analysis of copy number variants in 11 pairs of monozygotic twins with neurofibromatosis type 1. Am J Med Genet A. 2017:173:647-53.

9. Hernández-Martín A, Torrelo A. Rasopathies: developmental disorders that predispose to cancer and skin manifestations. Actas Dermosifiliogr. 2011;102:402-16.

10. Vaucheret Paz E, López Ballent A, Puga C, García Basalo MJ, Baliarda $\mathrm{F}$, Ekonen $\mathrm{C}$, et al. Cognitive profile and disorders affecting higher brain functions in paediatric patients with neurofibromatosis type 1. Neurologia. 2017:17:30148-2.

11. Cammarata-Scalisi F, Lacruz-Rengel MA, Stock F, Vidales C, Callea M. Aspectos clínicos y genéticos del complejo esclerosis tuberosa. Arch Venez Puer Ped. 2017;80:27-33.

12. Cammarata-Scalisi F, Vidales Moreno C, Stock F, Avendaño A, Araque D, Lacruz-Rengel MA, et al. Two novel mutations in the TSC2 gene causing severe phenotype in nervous system and skin in a patient with tuberous sclerosis complex. J Eur Acad Dermatol Venereol. 2017;32:e243-5

13. Wang YY, Pang LY, Ma SF, Zhang MN, Liu LY, Zou LP. Epilepsy may be the major risk factor of mental retardation in children with tuberous sclerosis: A retrospective cohort study. Epilepsy Behav. 2017;77:13-8.

14. Avgeris S, Fostira F, Vagena A, Ninios Y, Delimitsou A, Vodicka R, et al Mutational analysis of TSC1 and TSC2 genes in tuberous sclerosis complex patients from Greece. Sci Rep. 2017;7:16697.

15. Monteiro T, Garrido C, Pina S, Chorão R, Carrilho I, Figueiroa S, et al Tuberous sclerosis: clinical characteristics and their relationship to genotype/phenotype. An Pediatr (Barc). 2014;81:289-96.

16. Capal JK, Horn PS, Murray DS, Byars AW, Bing NM, Kent B, et al. Utility of the Autism Observation Scale for Infants in early identification of autism in tuberous sclerosis complex. Pediatr Neurol. 2017;75:80-6.

17. Papadopoulou A, Dinopoulos A, Koutsodontis G, Pons R, Vorgia P, Koute V, et al. Screening for TSC1 and TSC2 mutations using NGS in Greek children with tuberous sclerosis syndrome. Eur J Paediatr Neurol. 2018;22:419-26.
18. Northrup H, Krueger DA; International Tuberous Sclerosis Complex Consensus Group. Tuberous sclerosis complex diagnostic criteria update: recommendations of the 2012 International Tuberous Sclerosis Complex Consensus Conference. Pediatr Neurol. 2013;49:243-54.

19. DeBella K, Szudek J, Friedman JM. Use of the national institutes of health criteria for diagnosis of neurofibromatosis 1 in children. Pediatrics. 2000;105:608-14

20. Hernández-Martín A, Duat-Rodríguez A. An update on neurofibromatosis type 1: Not just café-au-lait spots, freckling, and neurofibromas. An update. part I. Dermatological clinical criteria diagnostic of the disease. Actas Dermosifiliogr. 2016;107:454-64.

21. Blakeley JO, Bakker A, Barker A, Clapp W, Ferner R, Fisher MJ, et al. The path forward: 2015 International Children's Tumor Foundation conference on neurofibromatosis type 1, type 2, and schwannomatosis. Am J Med Genet A. 2017;173:1714-21.

22. Hirabaru K, Matsuo M. Neurological comorbidity in children with neurofibromatosis type 1. Pediatr Int. 2017;60:70-5. doi: 10.1111/ped.13388.

23. Bilder DA, Bakian AV, Stevenson DA, Carbone PS, Cunniff C Goodman $A B$, et al. Brief report: the prevalence of neurofibromatosis type 1 among children with autism spectrum disorder identified by the autism and developmental disabilities monitoring network. J Autism Dev Disord. 2016;46:3369-76.

24. Flotats-Bastardas M, Ebrahimi-Fakhari D, Gortner L, Poryo M, Zemlin M, Macaya-Ruiz A, et al. Diagnosis and treatment of tuberous sclerosis manifestations in children: A multicenter study. Neuropediatrics. 2018;49:193-9.

25. Davis PE, Filip-Dhima R, Sideridis G, Peters JM, Au KS, Northrup H, et al. Presentation and diagnosis of tuberous sclerosis complex in infants. Pediatrics. 2017;140:e20164040. doi: 10.1542/peds.2016-4040.

26. Macaya A, Torra R; en representación del Grupo Español Multidisciplinar de expertos en Complejo Esclerosis Tuberosa (GEM-CET). Recommendations for the multidisciplinary management of tuberous sclerosis complex. Med Clin (Barc). 2016;147:211-6. 